\title{
Serum Biochemical Reference Values for Adult and Non-adult Chinese Alligators during the Deep and Late Hibernation Periods
}

\author{
Mingyan Tang', Rongmei Fei', Yilong Wu' \& Yongkang Zhou ${ }^{2}$
}

\begin{abstract}
Background: The Chinese alligator (Alligator sinensis) is a critically endangered species. Due to the rapid growth of the captive population, the susceptibility to disease during the recovery period after winter hibernation, especially in young alligators, have detrimentally affected Chinese alligator populations. Serum biochemistry, which relates to metabolism, nutritional status and disease, is enormously helpful in evaluating physical conditions in reptile. Many studies have reported the serum biochemical reference values of various reptilian species, including several crocodilians. However, reference values for Chinese alligators have not yet been reported. For captive Chinese alligators, hibernation is a crucial period because winter management has a direct influence on the survival rate of juveniles and the reproduction rate of adults. The main object of the present study refore was to measure the serum biochemical values of captive Chinese alligators during hibernation.

Materials, Methods \& Results: As such, this study investigates the serum biochemistry as a factor of age and hibernation stage. During the deep and late hibernation periods blood samples were drawn from 30 healthy captive Chinese alligators (adults, sub-adults, and juveniles) at the Anhui Research Center of Chinese Alligator Reproduction (ARCCAR). Serum biochemical measurements were performed using an automated biochemical analyzer and compared based on the age group and hibernation stage via two-way ANOVA. During late hibernation, serum lactate dehydrogenase, alkaline phosphatase, and aspartate aminotransferase activity increased in all age groups in comparison to that in deep hibernation, while the concentration of calcium decreased. Meanwhile, the concentration of serum phosphorus, uric acid, total protein, and globulin in sub-adults and juveniles considerably increased in comparison to that in deep hibernation, while cholesterol and albumin declined. However, in adults only slight changes were noted. Based on comprehensive statistical analysis, our results indicate that sub-adults and juveniles are at risk of developing renal disease during artificial hibernation.

Discussion: Chinese alligators, especially sub-adults and juveniles, are particularly vulnerable to disease when they wake from hibernation. They often display symptoms such as depression, anorexia, lethargy, sluggish movement, slow, incremental weight gain, progressive muscle wasting, and even death. The high rate of morbidity in non-adult Chinese alligators may be associated with the high density of UA and other changes in multiple biochemical markers that occur during late hibernation. These altered serum biochemical profiles may indicate kidney damage. One of the most common diseases among reptiles is nephropathy, the symptoms of which are non-specific and tend to agree with those observed post-hibernation. In summary, this study has reported the serum biochemical values of Chinese alligators of varying ages in the deep and late hibernation phases. Based on statistical analyses, interesting differences between the serum biochemical values of adults and non-adults during the deep and late hibernation have been found. The observed changes suggest that, under an artificial hibernation environment, the kidneys of sub-adults and juveniles may become impaired. We believe that the data reported in this study will provide clinical guidance to facilitate more appropriate artificial wintering conditions for Chinese alligators, and assist the breeding and management of these reptiles, as well as disease prevention, during hibernation and recovery.
\end{abstract}

Keywords: serum biochemistry, Chinese alligators, hibernation, disease susceptibility. 


\section{INTRODUCTION}

The Chinese alligator (Alligator sinensis) is one of the most endangered crocodilian species in the world and is indigenous to China [1-3]. In the 1970s the number of wild Chinese alligators decreased rapidly to no more than 120 due to further decline of their natural habitats $[7,15,16,18]$. The Anhui Research Center of Chinese Alligator Reproduction (ARCCAR) was established by the Chinese government in 1979, which focused on captive breeding. However, due to the rapid growth of the captive population, the susceptibility to disease during the recovery period after winter hibernation, especially in young alligators, have detrimentally affected Chinese alligator populations [22].

Serum biochemistry, which relates to metabolism, nutritional status and disease, is enormously helpful in evaluating physical conditions in reptile. Many studies have reported the serum biochemical reference values of various reptilian species, including several crocodilians [7,9,10,12]. However, reference values for Chinese alligators have not yet been reported. For captive Chinese alligators, hibernation is a crucial period because winter management has a direct influence on the survival rate of juveniles and the reproduction rate of adults [21]. Therefore, measuring the serum biochemical values of captive Chinese alligators during hibernation is particularly valuable.

The puropse of the current study was to obtain and analyze serum biochemical reference values of healthy captive Chinese alligators over three age groups (adults, sub-adults, and juveniles) at two hibernation stages, deep hibernation and late hibernation.

\section{MATERIALS AND METHODS}

\section{Animals}

November to early April is the typical hibernation of Chinese alligators. According to the individual alligator response and ambient temperature, hibernation is divided into three stages: early hibernation, from the onset of hibernation to late November (when Chinese alligators are still sensitive to their surroundings and the ambient temperature is around $14^{\circ} \mathrm{C}$ ); deep hibernation, from late November to mid-February (when Chinese alligators completely lose consciousness and the ambient temperature is 7 to $8^{\circ} \mathrm{C}$ ), and late hibernation (when Chinese alligators become sensitive to their surroundings again and the ambient temperature is around $14^{\circ} \mathrm{C}$ ) [16].
The animals used in this study were all hibernated indoors at the ARCCAR ( $\left.30^{\circ} 54 \mathrm{~N}, 118^{\circ} 46 \mathrm{E}\right)$. For the purposes of hibernation, Chinese alligators were placed in different rooms based on age. Thirty Chinese alligators were then selected for the study of deep (samples collected in late December) and late hibernation (samples collected in early March). These selected reptiles were divided into three age groups: adults (7-10 years), sub-adults (4-6 years), and juveniles (2-3 years), with 10 alligators in each age group. For the adult and sub-adult groups, $50 \%$ were male and $50 \%$ were female. The gender of the alligators was determined by cloaca palpation; however, establishing the gender of juveniles was not possible. Each individual was examined and no clinical signs of trauma were observed. The average indoor temperature was approximately $7^{\circ} \mathrm{C}$ to $8^{\circ} \mathrm{C}$ during deep hibernation and approximately $10^{\circ} \mathrm{C}$ to $13^{\circ} \mathrm{C}$ during late hibernation.

\section{Sample collection}

Each alligator was moved from the wintering room and manually restrained. Blood $(4 \mathrm{~mL})$ was drawn from the ventral coccygeal vein using a 6 $\mathrm{mL}$ additive-free vacuum blood collection tube (BD Vacutainer $\left.^{\circledR}\right)^{1}$ with a 20 gauge needle. After sample collection, the weight and length of each alligator was measured. Blood samples were stored in the refrigerator and assessed within $6 \mathrm{~h}$. Serum was obtained via centrifugation of the blood samples at 3,000 rpm for $10 \mathrm{~min}$ in an Eppendorf Centrifuge $5430^{2}$ and stored at $-20^{\circ} \mathrm{C}$ for 20 days to prepare the serum samples for subsequent automatic biochemical analyses (SINNOWA D240) ${ }^{3}$.

The following biochemical variables were tested: total protein (TP), albumin (ALB), globulin (GLO), albumin/globulin (A/G), uric acid (UA), urea, gammaglutamyltransferase (GGT), creatinine (Cre), cholesterol $(\mathrm{CHO})$, triglycerides (TG), calcium $(\mathrm{Ca})$ and phosphorus (P) concentrations, and alkaline phosphatase (ALP), alanine aminotransferase (ALT), aspartate aminotransferase (AST), lactate dehydrogenase (LDH), amylase (AMY) and creatine kinase (CK) activity.

\section{Statistical analysis}

All statistical analyses were performed using SPSS for Windows. Biochemical variables are reported as the mean $\pm \mathrm{SD}$ and in ranges (minimum-maximum values). Data distributions were assessed using normalcy and equal variance tests. Data from the Chinese 
alligators were statistically compared based on age (adults, sub-adults, and juveniles) and the hibernation stage (deep hibernation and late hibernation) as follows: for data that were normally distributed, analysis was performed using two-way analysis of variance (ANOVA) and significant differences were assessed using Tukey's test; for data that were not normally distributed, comparisons were made using the MannWhitney U-test. Results were considered significant at $P<0.05$ and extremely significant at $P<0.01$.

\section{RESULTS}

There were no statistically significant differences between the bodyweight and length of alligators in all age groups for the two hibernation periods $(P>$ 0.05 , Table 1) and the results of the plasma biochemistry analyses are presented in Table 2 .

During deep hibernation, the serum cholesterol concentration in juveniles was significantly higher $(P<0.05)$ than in adults, while the UA concentration was significantly lower $(P<0.05)$ than in adults. In addition, the serum globulin concentration in juveniles was significantly lower $(P<0.05)$ than in sub-adults and this decrease was even more evident compared to adults $(P<0.01)$.

During late hibernation the serum biochemistry results was noted to change considerably in all age groups. For example, the serum LDH and ALP activities were notably increased $(P<0.01)$ in comparison to that in deep hibernation, as was the AST activity $(P<0.05)$, while the serum calcium concentration significantly decreased in adults, sub-adults, and juveniles $(P<0.01)$ compared to that in deep hibernation. Meanwhile, in sub-adults and juveniles, the concentration of phosphorus, UA, total protein, and globulin increased significantly $(P<0.01)$, while albumin and cholesterol concentrations decreased $(P<0.01)$. Interestingly, these parameters remained unchanged in adults.

Table 1. The body length and weight of Chinese alligators (adults, sub-adults, and juveniles) during deep and late hibernation.

\begin{tabular}{|c|c|c|c|c|c|c|c|c|}
\hline \multirow{3}{*}{ Age group } & \multicolumn{4}{|c|}{ Deep hibernation } & \multicolumn{4}{|c|}{ Late hibernation } \\
\hline & \multicolumn{2}{|c|}{ Body length (m) } & \multicolumn{2}{|c|}{ Body weight (kg) } & \multicolumn{2}{|c|}{ Body length (m) } & \multicolumn{2}{|c|}{ Body weight (kg) } \\
\hline & Mean \pm SD & Range & Mean \pm SD & Range & Mean \pm SD & Range & Mean \pm SD & Range \\
\hline Adults $(n=10)$ & $1.56 \pm 0.10$ & $1.45-1.7$ & $14.89 \pm 3.13$ & $10.45-19.57$ & $1.56 \pm 0.08$ & $1.48-1.64$ & $15.03 \pm 2.25$ & $11.5-17.5$ \\
\hline Sub-adults $(n=10)$ & $1.26 \pm 0.01$ & $1.12-1.32$ & $7.4 \pm 0.09$ & $5.52-8.47$ & $1.23 \pm 0.04$ & $1.14-1.31$ & $7.42 \pm 0.00$ & $5.85-8.3$ \\
\hline Juveniles $(n=10)$ & $1.05 \pm 0.01$ & $0.97-1.06$ & $4.12 \pm 0.16$ & $2.91-4.25$ & $1.00 \pm 0.05$ & $0.95-1.07$ & $4.19 \pm 0.65$ & $3.22-5.4$ \\
\hline
\end{tabular}

\section{DISCUSSION}

The serum biochemistry of reptiles can be influenced by various factors, such as gender, age, nutrition, physiological status, pathological conditions and seasonal variations. Some previous studies have reported that the activity of certain serum or plasma enzymes in other reptiles declines drastically in winter, when temperatures were lower $[5,6,17]$. As the Chinese alligators entered deep hibernation, the ambient temperatures were in the range of $7^{\circ} \mathrm{C}$ to $8^{\circ} \mathrm{C}$ and the alligators' metabolism slowed to the lowest level. As a consequence, alligators lay still, with their eyes closed, and exhibited hardly any reaction to stimulation; they were in a state of complete unconsciousness. As the alligators transitioned to late hibernation, the ambient temperatures rose to between $12^{\circ} \mathrm{C}$ and $14^{\circ} \mathrm{C}$ and the alligators became slightly sensitive to the surrounding environment. During late hibernation the serum biochemistry profiles of vary- ingly aged Chinese alligators changed enormously and analysis revealed that the activity of alkaline phosphatase (ALP), lactate dehydrogenase (LDH), and aspartate aminotransferase (AST) increased significantly in all age groups. This change is probably due to the more vigorous metabolic activity that Chinese alligators exhibit during late hibernation, as well as the higher ambient temperatures, in comparison to the deep hibernation stage. AST, LDH, and ALP are present in a wide variety of tissues, such as the liver, kidney and muscles. Their levels increase in relation to body metabolism and environmental temperature, as well as during hepatic or renal disease, or following muscle damage. In addition, hemolysis can lead lactate dehydrogenase (LDH) activity to rise. During our study, no clinical signs of muscle injury were found during exterior examination, and the blood samples taken from alligators showed no evidence of hemolysis. Thus, the rise in the serum biochemical 


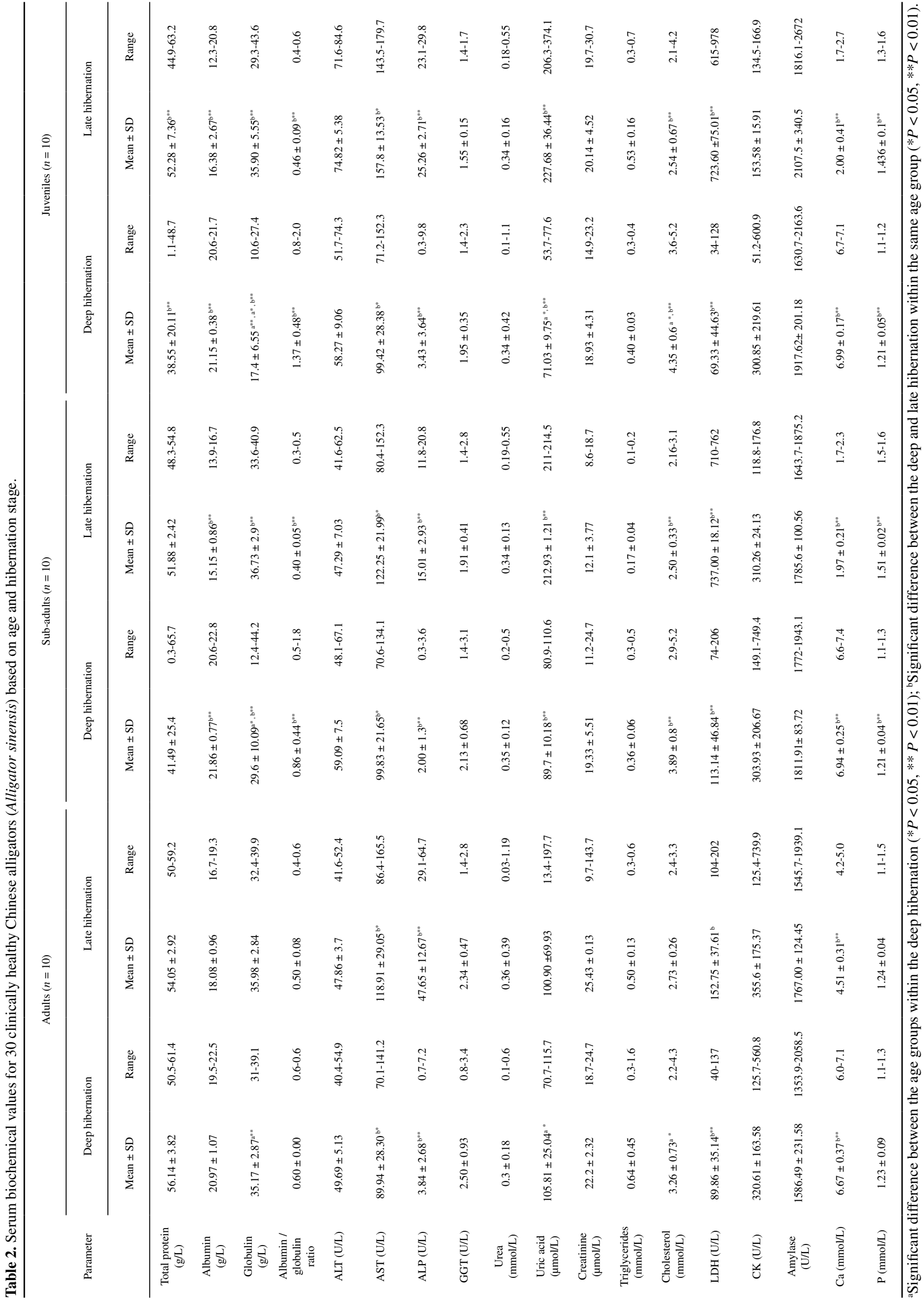


values of these enzymes is unlikely to be the result of muscle injury and hemolysis. During late hibernation, the activity of alkaline phosphatase (ALP) and lactate dehydrogenase (LDH) in all three age groups increased notably, especially in sub-adults and juveniles. There are two possible explanations for this: first, the increase may be considered to be a normal change that occurs during hibernation from the deep to the late stage; alternatively, the increase may be considered to be beyond the normal range and due to hepatic or renal disease. To establish the true cause of these increases in enzyme activity, further analysis is required that also considers other serum biochemical values.

As such, we also examined the levels of UA during late hibernation and found that, in sub-adults and juveniles, the concentration rose sharply from $89.7 \mu \mathrm{mol} / \mathrm{L}$ and $71.3 \mu \mathrm{mol} / \mathrm{L}$, respectively, during deep hibernation to $212.9 \mu \mathrm{mol} / \mathrm{L}$ and $227.6 \mu \mathrm{mol} / \mathrm{L}$, respectively, in late hibernation. Furthermore, the concentration of phosphorus and globulin (GLO) was also noted to increase significantly in late hibernation for sub-adults and juveniles, while the ALB concentration decreased substantially. However, these changes were not observed in adult alligators.

It has been reported that chronic renal disease in reptiles is associated with the elevation of plasma phosphorus concentrations, alternation of the calcium: phosphorus ratio, an increase in plasma UA concentrations $[4,13,14,20]$, and the notable decrease of albumin caused by its quantitative loss from the damaged kidney. As such, these changes, as well as immune system hyperactivity and elevation of immune globulin concentrations, provide supporting evidence for the diagnosis of possible renal disease in reptiles. Considering our data, it may be concluded that sub-adults and juveniles may suffer renal injury or insufficiency when contained within an artificial hibernation environment. The causes of these pathological changes may be the limited living conditions of the artificial winter environment compared to natural winter conditions. In the natural environment Chinese alligators hibernate in caves, which have well-hidden entrances, meandering tunnels, and spacious areas, where there are platforms and pools. Thus, the caves provide insulation, humidity, and protection from external stressors. Furthermore, natural light in hibernating caves has no diurnal variation, and males and females dwell separately on clay surfaces in small spaces without interference. In contrast, captive Chinese alligators hibernate in wintering rooms in which the indoor temperature is maintained at $8-14^{\circ} \mathrm{C}$ by heaters, light intensity varies with time, and males and females hibernate on concrete in mixed, open-space rooms with a certain degree of human disturbance. It must be noted however that, during late hibernation, the concentration of UA, phosphorus, albumin, and globulin barely fluctuated in adults. Thus, it can be assumed that the physiological function of adult alligators differs from that in sub-adults and juveniles. This may be due to adults being more tolerant of the environment and having higher physiological regulation capabilities. Considering the activity of ALP and LDH increases in sub-adults and juveniles compared to adults, the liver/kidney dysfunction may result from the sub-optimal conditions of artificial hibernation, which are not sufficiently akin to natural conditions.

Chinese alligators, especially sub-adults and juveniles, are particularly vulnerable to disease when they wake from hibernation. They often display symptoms such as depression, anorexia, lethargy, sluggish movement, slow, incremental weight gain, progressive muscle wasting, and even death. The high rate of morbidity in non-adult Chinese alligators may be associated with the high density of UA and other changes in multiple biochemical markers that occur during late hibernation. These altered serum biochemical profiles may indicate kidney damage. One of the most common diseases among reptiles is nephropathy $[8,15]$, the symptoms of which are non-specific and tend to agree with those observed post-hibernation.

\section{CONCLUSIONS}

In conclusion, this study has reported the serum biochemical values of Chinese alligators of varying ages in the deep and late hibernation phases. Based on statistical analyses, interesting differences between the serum biochemical values of adults and non-adults during the deep and late hibernation have been found. The observed changes suggest that, under an artificial hibernation environment, the kidneys of sub-adults and juveniles may become impaired.

We believe that the data reported in this study will provide clinical guidance to facilitate more appropriate artificial wintering conditions for Chinese alligators, and assist the breeding and management of these reptiles, as well as disease prevention, during hibernation and recovery. As an example, preventative measures can be taken to accelerate the excretion of UA from non-adults after waking, which may reduce the incidence of nephropathy in this protected species. 
MANUFACTURERS

${ }^{1}$ Becton, Dickinson and Company. Franklin Lakes, NJ, USA.

${ }^{2}$ Eppendorf. Hamburg, Germany.

${ }^{3}$ Sinnowa Medical Science \& Technology Co. Ltda. Nanjing, Jiangsu, China.

Acknowledgments. The authors thank the staff the Anhui Research Center of Chinese Alligator Reproduction (ARCCAR) for their assistance. This project was supported by a grant from the Priority Academic Program Development of Jiangsu Higher Education Institutions.

Ethical approval. This experiment was approved and performed under the guidelines of Ethics Committee for Animal Use of Nanjing Agricultural University.

Declaration of interest. The authors declare they have no conflicts of interest.

\section{REFERENCES}

1 Chen B.H., Hua T.M., Wu X.B. \& Wang C. 2003. Research on the Chinese alligator. Shanghai: Shanghai Science and Technology Press, pp.221-268.

2 Chen B.H. 1990. The past and present situation of the Chinese alligator. Asiatic Herpetological Research. 3: 129-136.

3 Ding Y.Z. \& Wang X.M. 2004. Factors influencing the population status of wild Chinese alligators (Alligator sinensis). Biodiversity Science. 12(3): 324-332.

4 Ding Y.Z., Wang X.M., He L.J. \& Shao M. 2001. Study on the current population and habitat of the wild Chinese alligator (Alligator sinensis). Biodiversity Science. 9(2): 102-108.

5 Divers S., Redmayne G. \& Aves E. 1996. Haematological and biochemical values of 10 green iguanas (Iguana iguana). Veterinary Record. 138(9): 203-205.

6 Divers S.J. 1997. Clinician's approach to renal disease in lizards. Proceedings of of the 4th Annual Association Reptilian and Amphibian Veterinarians Conference (Houston, USA). pp.5-11.

7 Lovely C.J., Pittman J.M. \& Leslie A.J. 2007. Normal haematology and blood biochemistry of wild Nile crocodiles (Crocodylus niloticus) in the Okavango Delta, Botswana. Journal of the South African Veterinary Association. 78(3): 137-144.

8 Mateo M.R., Roberts E.D. \& Enright F.M. 1984. Morphologic, cytochemical, and functional studies of peripheral blood cells of young healthy American alligators (Alligator mississippiensis). American Journal of Veterinary Research. 45(5): 1046-1053.

9 Millan J., Janmaat A., Richardson K., Chambers L.K. \& Fomiatti K.R. 1997. Reference ranges for biochemical and haematological values in farmed saltwater crocodile (Crocodylus porosus) yearlings. Australian Veterinary Journal. 75(11): 814-817.

10 Miller H.A. 1998. Urinary diseases of reptiles: pathophysiology and diagnosis. Seminars in Avian and Exotic Pet Medicine. WB Saunders. 7(2): 93-103

11 Reavill D.R. \& Schmidt R.E. 2010. Urinary Tract Diseases of Reptiles. Journal of Exotic Pet Medicine. 19(4): 280289.

12 Rossini M., García G., Rojas J. \& Zerpaet H. 2011. Hematologic and serum biochemical reference values for the wild Spectacled Caiman, Caiman crocodilus crocodilus, from the Venezuelan plains. Veterinary Clinical Pathology. 40(3): 374-379.

13 Selleri P. \& Hernandez-Divers S.J. 2006. Renal diseases of reptiles. Veterinary Clinics of North America: Exotic Animal Practice. 9(1): 161-174.

14 Silva L., Riani-Costa C., Ramos P. \& Takahira R. 2011. Seasonal influence on biochemical profile and serum protein electrophoresis for Boa constrictor amarali in captivity. Brazilian Journal of Biology. 71(2): 517-520.

15 Thorbjarnarson J. \& Wang X.M. 1999. The conservation status of the Chinese alligator. Oryx. 33(2): 152-159.

16 Thorbjarnarson J., Wang X.M., Ming S., He L. \& Ding Y. 2002. Wild populations of the Chinese alligator approach extinction. Biological Conservation. 103(1): 93-102.

17 Wang R.P., Zhou Y.J. \& Wang C.L. 1998. The relationship between the environment temperature and the life habits of the Chinese alligator (Alligator sinensis). Chinese Journal of Zoology. 33(2): 33-35.

18 Watanabe M.E. 1983. The Chinese Alligator: is farming the last hope? Oryx. 17(4): 176-181.

19 Wilkinson R., Mcarthur S. \& Meyer J. 2004. Clinical pathology. In: Mcarthur S., Wilkinson R. \& Meyer J. (Eds). Medicine and surgery of tortoises and turtles. Oxford: Blackwell Publishing Ltd., pp. 141-186. 
M. Tang, R. Fei, Y. Wu \& Y. Zhou. 2016. Serum Biochemical Reference Values for Adult and Non-adult Chinese Alligators during the Deep and Late Hibernation Periods. Acta Scientiae Veterinariae. 44: 1416.

20 Xia T.S., Zhou K.H. \& Zhu J.L. 2006. Influence of Hibernant Temperature on Reproduction of Chinese Alligator. Sichuan Journal of Zoology. 25(2): 398-399.

21 Zaias J., Norton T., Fickel A., Spratt J., Altman N.H. \& Cray C. 2006. Biochemical and hematologic values for 18 clinically healthy radiated tortoises (Geochelone radiata) on St Catherines Island, Georgia. Veterinary Clinical Pathology. 35(3): 321-325.

22 Zhou Y.K., Yu B.F. \& Wu X.B. 2012. Present status of protection on Chinese alligator's population and habitat. Chinese Journal of Zoology. 47(1): 133-136. 